\title{
Efeito da frequência e amplitude de vibração sobre a derriça de frutos de café
}

\author{
$\overline{\text { Fábio L. Santos }{ }^{1}, \text { Daniel M. de Queiroz }{ }^{2} \text {, Francisco de A. de C. Pinto }{ }^{2} \& \text { Ricardo C. de Resende }{ }^{2}}$
}

RESUMO

Parâmetros de qual idade podem alterar significativamente o preço do café; entretanto, a colheita seletiva dos frutos é frequentemente associada à boa qualidade do produto, procedimento que pode ser realizado por meio de vibrações mecânicas. Desta forma, o estudo e a avaliação do efeito localizado de fatores como frequência, amplitude e grau de maturação dos frutos, são fundamentais para construção de máquinas adequadas a este tipo de prática. 0 trabalho foi desenvolvido com o objetivo de se avaliar os fatores frequência, amplitude, grau de maturação, direção de excitação e número de frutos por pedúnculo, na eficiência de derriça. Para a realização dos ensaios de vibração em laboratório utilizou-se uma máquina vibradora eletromagnética. Amplitudes na faixa de 3,75 a 7,50 mm e frequências na faixa de 13,33 a 26,67 Hz foram testadas para a variedade Mundo Novo, em que os resultados mostraram que a frequência de $26,67 \mathrm{~Hz}$ apresentou uma eficiência maior de derriça para os frutos cereja e a amplitude de 7,5 mm indicou maior eficiência de derriça. Verificou-se, também, que o número de frutos por pedúnculo influencia a eficiência de derriça dos frutos de café da variedade Mundo Novo.

Palavras-chave: vibrações mecânicas, mecanização, colheita, grau de maturação

\section{Frequency and amplitude of vibration on coffee harvesting}

\begin{abstract}
Q uality parameters influence directy the coffee price. However, selective coffee harvesting is frequently associated to good quality of this product. This procedure can be performed by mechanical vibration. Therefore, the study of the frequency and amplitude parameters is important for the design of a specific harvesting machine. The objective of this work was to evaluate the effect of the frequency and amplitude of vibration, the coffee variety and the ripeness condition of the fruits upon the harvesting efficiency. The vibration tests were done in laboratory using an electromagnetic shaker. The tests were done using amplitudes in the range of 3.75 to $7.50 \mathrm{~mm}$ and frequencies in the range of 13.33 to $16.67 \mathrm{~Hz}$. Branches of coffee plants of M undo N ovo variety were tested. The highest harvesting efficiency was obtained when using the $26.67 \mathrm{~Hz}$ frequency of vibration. The highest harvesting efficiency was obtained when an amplitude of $7.5 \mathrm{~mm}$ was used. It was also observed that the number of fruits per stem influences the harvesting efficiency of the coffee fruits of the Mundo N ovo variety.
\end{abstract}

Key words: mechanical vibrations, mechanization, harvesting, ripeness condition

1 FCA/U FGD, Rodovia Dourados à Itahum km 12, Caixa Postal 533, CEP 79804-970 Dourados, MS. Fone: (67) 3411-3810. E-mail: fabiosantos@ufgd.edu.br 2 DEA/UFV, Av. Peter Henry Rolfs s/nº, Campus Universitário, CEP 36570-000 Viçosa, MG. Fone: (31) 3899-2729. E-mail(s): queiroz@ufv.br; facpinto@ufv.br; ricardocapucio@ufv.br 


\section{INTRODUÇÃO}

A colheita pode ser considerada uma das mais complexas e onerosas etapas do ciclo de produção do café, devido à grande demanda de mão-de-obra e por estar associada ao índice de qualidade do produto (Oliveira et al., 2007).

O café é um produto cujo preço está diretamente relacionado a parâmetros de qualidade, podendo as perdas financeiras variar de 10 a $20 \%$ quanto ao aspecto do produto, $40 \%$ em função da bebida e até $60 \%$ para café de mau aspecto e apresentando bebida ruim (Filgueiras, 2001).

A colheita do café pode ser descrita em três etapas: a primeira etapa consiste da derriça ou catação, a segunda é composta pela varrição e/ou pelo recolhimento e a terceira se refere à abanação dos frutos. Durante a etapa de derriça a árvore pode ser colhida de uma única vez ou de forma seletiva, em que somente os frutos maduros são colhidos (Souza, 2004).

A derriça total dos frutos presentes na árvore pode proporcionar perda de qualidade da bebida do café, caso não sejam tomadas providências no sentido de isolar os frutos em cada estádio de maturação e de eliminar as impurezas de colheita, seja na fase relativa à pós-colheita ou mesmo durante a fase de beneficiamento do produto (Oliveira et al., 2007).

A colheita mecanizada de frutos de café tem sido realizada de forma eficiente, por meio de vibrações mecânicas. Tal princípio se baseia na transferência de energia vibracional para o sistema fruto-pedúnculo, a qual promove o desprendimento dos frutos do cafeeiro (Srivastava et al., 1996; Ciro, 2001).

Derriçadoras portáteis, que utilizam o princípio de vibrações mecânicas, apresentam desempenho operacional superior ao obtido pela colheita manual de café (Barbosa et al., 2005; Tascon et al., 2005). Adicionalmente, Souza et al. (2006) verificaram que um desempenho operacional melhor de derriçadoras portáteis, é obtido quando se empregam duas máquinas simultaneamente, em uma mesma linha de plantio.

Segundo Souza (2004), máquinas que utilizam vibrações mecânicas como princípio de funcionamento, podem ser empregadas na colheita seletiva de frutos e, desta forma, o conhecimento do comportamento dinâmico desses sistemas é fundamental para o sucesso de tal procedimento. Dentre as principais características dinâmicas se destacam as frequências e amplitudes de vibrações forçadas mais adequadas ao processo de derriça dos frutos (Souza et al., 2002; Sessiz \& Özcan, 2006; Castro-García et al., 2008). Assim, para projetos de máquinas destinadas à colheita seletiva de café é necessário o conhecimento da interação dos vários fatores que influenciam este procedimento, razão por que este trabalho foi desenvolvido com o objetivo de analisar o efeito localizado de fatores como frequência e amplitude de vibração, grau de maturação, número de frutos por pedúnculo e direção de vibração sobre os sistemas fruto-pedúnculo dos frutos de café, sob eficiência de derriça, para a variedade Mundo Novo.

\section{MATERIAL E MÉTODOS}

Este trabalho foi executado no Laboratório de Projetos de Máquinas e Visão Artificial (PROVISAGRO) do Departamento de Engenharia Agrícola da Universidade Federal de Viçosa utilizando-se um sistema produzido pela LDS (Ling Dynamic Systems) composto por um gerador de sinais COMET $_{\text {USB }}$ da marca Dactron, um amplificador PA100E-CE e uma máquina vibradora eletromagnética modelo V-406.

$\mathrm{Na}$ Tabela 1 se apresentam as características técnicas da máquina vibradora eletromagnética, a qual foi montada em um berço metálico, o que possibilitou a realização de ensaios de vibração nos sentidos longitudinal e transversal em relação a um ramo plagiotrópico de café.

Tabela 1. Características técnicas da máquina vibradora eletromagnética

\begin{tabular}{lc}
\hline Faixa dinâmica de trabalho $(\mathrm{Hz})$ & $5-9000$ \\
Carga máxima $(\mathrm{N})$ & 198 \\
Deslocamento máximo da base móvel $(\mathrm{mm})$ & 17,6 \\
Aceleração máxima $(\mathrm{g})$ & 100 \\
\hline
\end{tabular}

O gerador de sinais COMET $_{\text {USB }}$ da marca Dactron, por meio de um programa específico fornecido pela LDS, pode ser ajustado para a geração de sinais senoidais, aleatórios e de impacto, os quais são amplificados pelo amplificador PA100E$\mathrm{CE}$, antes de serem enviados à máquina vibradora. Optou-se, para o presente trabalho, pela utilização de sinais senoidais, uma vez que este tipo de sinal representa a forma de excitação mais comum encontrada nas máquinas colhedoras de café.

A estrutura empregada para a fixação dos ramos plagiotrópicos de café à máquina vibradora foi constituída por uma base rígida, na qual se adaptou um mandril que possibilitou o engastamento do ramo ao sistema, sem qualquer dano à sua estrutura. Um transdutor piezoelétrico de aceleração, ou acelerômetro, empregado no controle do sistema, permitiu testar diferentes frequências e amplitudes de vibração dos ramos de café, por meio do deslocamento da base móvel da máquina vibradora.

O sistema exposto foi utilizado para executar um experimento segundo o delineamento inteiramente casualizado com três repetições no esquema fatorial. Neste experimento foram avaliados, para a variedade Mundo Novo, os fatores frequência, direção e amplitude de vibração; graus de maturação e número de frutos por pedúnculo com relação ao efeito pontual que proporcionam na eficiência de derriça do sistema fruto-pedúnculo. Os níveis dos fatores avaliados neste experimento são apresentados na Tabela 2 .

Tabela 2. Níveis avaliados nos testes de vibração para determinação da eficiência de derriça

\begin{tabular}{lc}
\hline Frequência $(\mathrm{Hz})$ & 13,$33 ; 16,67 ; 20,00 ; 23,33 ; 26,67$ \\
Amplitude $(\mathrm{mm})$ & 3,$75 ; 5,0 ; 6,25 ; 7,50$ \\
Grau de maturação & verde; verdoengo; cereja; passa \\
Frutos por pedúnculo & $1 ; 2$ \\
Direção de vibração & Iongitudinal; transversal \\
\hline
\end{tabular}


A eficiência de derriça foi calculada, após a realização do ensaio de derriça por vibrações mecânicas, através da divisão do número de frutos derriçados pelo número total de frutos presentes no ramo, antes da realização do ensaio.

Os ramos utilizados neste experimento foram coletados de forma aleatória, em uma área experimental, localizada no campus da Universidade Federal de Viçosa; após a coleta, os ramos foram cortados em segmentos de cinco centímetros e classificados de acordo com o grau de maturação dos frutos e com o número de frutos por pedúnculo.

Os valores de eficiência de derriça obtidos com a realização deste experimento foram, inicialmente, submetidos a análise de variância; adicionalmente, os fatores qualitativos tiveram suas médias comparadas pelo teste de Tukey; por outro lado, os efeitos dos fatores quantitativos foram estudados por análise de regressão. Todas as análises estatísticas foram realizadas utilizando-se programa computacional SAS versão 8.0 considerando-se o nível de $5 \%$ de significância.

\section{RESULTADOS E DISCUSSÃO}

Apresenta-se, na Tabela 3, o resultado da análise de variância para a eficiência de derriça obtida a partir de ramos coletados na variedade Mundo Novo. Pode-se observar que todas as interações duplas entre os fatores avaliados neste experimento foram significativas a $5 \%$ de probabilidade. $\mathrm{O}$ desdobramento do efeito dos fatores de cada uma dessas interações é apresentado a seguir.

Tabela 3. Análise de variância para a eficiência da derriça de ramos coletados na variedade Mundo Novo

\begin{tabular}{lcrrcc}
\hline \multicolumn{1}{c}{ FV } & GL & \multicolumn{1}{c}{ SQ } & \multicolumn{1}{c}{ QM } & F & P-valor \\
Frequência (F) & 4 & 224567,71 & 56141,93 & - & - \\
Maturação (M) & 3 & 74424,48 & 24808,16 & - & - \\
Direção (D) & 1 & 1898,44 & 1898,44 & - & - \\
№ de frutos (N) & 1 & 23502,60 & 23502,60 & - & - \\
Amplitude (A) & 3 & 173570,31 & 57856,77 & - & - \\
F XA & 12 & 83682,29 & 6973,52 & $9,61^{*}$ & $<0,001$ \\
F XM & 12 & 32411,46 & 2700,95 & $3,72^{*}$ & $<0,001$ \\
FxN & 4 & 11588,54 & 2897,14 & $3,99^{*}$ & 0,0032 \\
M XA & 9 & 18356,77 & 2039,64 & $2,81^{*}$ & 0,0029 \\
F XD & 4 & 7307,29 & 1826,82 & $2,52^{*}$ & 0,0401 \\
Resíduo & 906 & 657750,00 & 725,99 & - & - \\
Total & 959 & 1309059,89 & - & - & - \\
\hline
\end{tabular}

* significativo a nível de $5 \%$ de probabilidade e ns não-significativo

Na Tabela 4 são apresentados os resultados obtidos do desdobramento da interação entre os fatores frequência e grau de maturação para a eficiência de derriça de ramos coletados na variedade Mundo Novo.

Pode-se constatar, na Tabela 4, que a diferença significativa foi detectada entre graus de maturação, nas frequências de $16,67,20,00,23,33$ e $26,67 \mathrm{~Hz}$; as médias da eficiência da derriça entre os graus de maturação nessas frequências foram comparadas pelo teste Tukey a 5\% de probabilidade cujos resultados obtidos são apresentados na Tabela 5.
Tabela 4. Resultados obtidos da análise de variância do desdobramento da interação entre os fatores frequência e grau de maturação com relação à eficiência da derriça, em ramos da variedade Mundo N ovo

\begin{tabular}{cccccc}
\hline FV & GL & SQ & QM & F & P-valor \\
Maturação/frequência $(13,33 \mathrm{~Hz})$ & 3 & 559,89 & 186,63 & 0,26 ns & 0,8563 \\
Maturação/frequência $(16,67 \mathrm{~Hz})$ & 3 & 19935,00 & 6644,96 & $9,15^{*}$ & $<0,001$ \\
Maturação/frequência $(20,00 \mathrm{~Hz})$ & 3 & 18490,00 & 6163,19 & $8,49 *$ & $<0,001$ \\
Maturação/frequência $(23,33 \mathrm{~Hz})$ & 3 & 25456,00 & 8485,24 & $11,69 *$ & $<0,001$ \\
Maturação/frequência $(26,67 \mathrm{~Hz})$ & 3 & 42396,00 & 14132,00 & $19,47 *$ & $<0,001$ \\
Resíduo & 906 & 657750,00 & 725,99 & - & - \\
\hline
\end{tabular}

* significativo ao nível de $5 \%$ de probabilidade e ${ }^{\mathrm{ns}}$ não-significativo

Tabela 5. Média da eficiência de derriça em função da frequência de vibração e do grau de maturação para ramos coletados na variedade Mundo Novo

\begin{tabular}{lcccc}
\hline \multirow{2}{*}{ Frequência (Hz) } & \multicolumn{4}{c}{ Grau de maturação } \\
\cline { 2 - 5 } 16,67 & Verde & Verdoengo & Cereja & Passa \\
20,00 & $0,00 \mathrm{~A}$ & $1,04 \mathrm{~A}$ & $4,17 \mathrm{~A}$ & $25,00 \mathrm{~B}$ \\
23,33 & $0,00 \mathrm{~A}$ & $18,75 \mathrm{~B}$ & $14,58 \mathrm{~B}$ & $27,08 \mathrm{C}$ \\
26,67 & $11,45 \mathrm{~A}$ & $31,25 \mathrm{~B}$ & $29,16 \mathrm{~B}$ & $43,75 \mathrm{C}$ \\
\hline
\end{tabular}

Médias seguidas de letras iguais não diferem entre si na mesma linha, segundo o teste de Tukey, a probabilidade de $5 \%$

Para a frequência de $16,67 \mathrm{~Hz}$ não houve diferença entre as médias de eficiência de derriça e entre os graus de maturação verde, verdoengo e cereja, tal como para as frequências de 20,00 e 23,33 Hz, em que não se constatou diferença significativa entre as médias para os graus de maturação verdoengo e cereja; entretanto, tais graus de maturação apresentaram eficiência de derriça superior ao grau de maturação verde. Os frutos verdoengos tiveram média idêntica da eficiência de derriça quando comparados com os frutos cereja para as frequências de 16,67, 20,00 e 23,33 Hz.

A frequência de vibração $26,67 \mathrm{~Hz}$ tendeu a apresentar maior média de eficiência de derriça para os frutos com grau de maturação desejado (cerejas e passas); para esta frequência, os frutos que ainda não estão no estágio apropriado de maturação tenderam a uma eficiência menor na derriça.

$\mathrm{O}$ efeito da frequência em cada um dos graus de maturação na eficiência da derriça em ramos coletados na variedade Mundo Novo, foi estudado por análise de regressão cujos resultados são apresentados na Tabela 6. Pode-se observar, em todos os graus de maturação, que a frequência de vibração teve efeito significativo a nível de $5 \%$ de probabilidade na eficiência da derriça, resultado que ressalta a importância

Tabela 6. Resultados da análise de regressão para o estudo do efeito da frequência de vibração em diferentes graus de maturação de frutos na eficiência da derriça de ramos coletados na variedade Mundo Novo

\begin{tabular}{ccccccccc}
\hline \multirow{2}{*}{ FV } & \multicolumn{2}{c}{ Verde } & \multicolumn{2}{c}{ Verdoengo } & \multicolumn{2}{c}{ Cereja } & \multicolumn{2}{c}{ Passa } \\
\cline { 2 - 9 } & GL & QM & GL & QM & GL & QM & GL & QM \\
Regressão & 1 & $15755,20^{*}$ & 1 & $51032,63^{*}$ & 1 & $93526,66 *$ & 1 & $76011,22^{*}$ \\
Resíduo & 906 & 725,99 & 906 & 725,99 & 906 & 725,99 & 906 & 725,99 \\
\hline *
\end{tabular}

* significativo ao nível de $5 \%$ de probabilidade 
que a frequência de vibração tem no processo de colheita de café por vibrações mecânicas.

As Eqs. 1, 2, 3 e 4 representam os modelos ajustados para o estudo do efeito da frequência de vibração nos diferentes graus de maturação dos frutos na eficiência de derriça para a variedade Mundo novo. Escolheram-se esses modelos com base no coeficiente de determinação, significância dos coeficientes de regressão e na falta de ajustamento.

$$
\begin{array}{ll}
\mathrm{E}_{\mathrm{d}-\mathrm{ve}}=-27,493+1,718 \mathrm{~F} & \left(\mathrm{R}^{2}=0,78\right) \\
\mathrm{E}_{\mathrm{d}-\mathrm{vd}}=-44,350+3,092 \mathrm{~F} & \left(\mathrm{R}^{2}=0,94\right) \\
\mathrm{E}_{\mathrm{d}-\mathrm{ce}}=-62,277+4,186 \mathrm{~F} & \left(\mathrm{R}^{2}=0,89\right) \\
\mathrm{E}_{\mathrm{d}-\mathrm{pa}}=-43,532+3,749 \mathrm{~F} & \left(\mathrm{R}^{2}=0,96\right)
\end{array}
$$

em que:

$E_{d-v e}, E_{d-v d}, E_{d-c e}$ e $E_{d-p a}$ - eficiência de derriça para os graus de maturação verde, verdoengo, cereja e passa

$\mathrm{F}=$ frequência de vibração

Na Figura 1A se apresenta o efeito da frequência de vibração em cada grau de maturação. Para todos os graus de maturação ocorreu aumento na eficiência de derriça na medida em que se aumentou a frequência de vibração; deve-se, contudo, ressaltar que a média de eficiência de derriça para os frutos cereja foi superior à média dos frutos verdoengos e verdes para frequências de vibração acima de $16,67 \mathrm{~Hz}$, o que é interessante para o procedimento de colheita seletiva; observa-se, ainda, que as maiores médias de eficiência de derriça foram obtidas para os frutos passas.

Apresenta-se, na Tabela 7, o desdobramento do efeito da amplitude de vibração em cada grau de maturação. Observouse que o efeito do fator amplitude foi significativo para todos os graus de maturação considerados, o que caracteriza sua importância, tal qual sua associação à frequência de vibração, no procedimento de derriça por vibrações mecânicas (Ciro, 2001).

Tabela 7. Resultados da análise de regressão para o estudo do efeito da amplitude em diferentes graus de maturação de frutos na eficiência da derriça de ramos coletados na variedade Mundo Novo

\begin{tabular}{lcccccccc}
\hline \multirow{2}{*}{ FV } & \multicolumn{2}{c}{ Verde } & \multicolumn{2}{c}{ Verdoengo } & \multicolumn{2}{c}{ Cereja } & \multicolumn{2}{c}{ Passa } \\
\cline { 2 - 10 } & GL & QM & GL & QM & GL & QM & GL & QM \\
Regressão & 1 & $13002,08^{*}$ & 1 & $59152,81 *$ & 1 & $52668,75 *$ & 1 & $58102,08 *$ \\
Resíduo & 906 & 725,99 & 906 & 725,99 & 906 & 725,99 & 906 & 725,99 \\
\hline
\end{tabular}

* significativo a nível de $5 \%$ de probabilidade

As Eqs. 5, 6, 7 e 8 representam os modelos escolhidos com base no coeficiente de determinação, na significância dos coeficientes e na falta de ajuste, para o estudo do efeito da amplitude de vibração em cada grau de maturação para a variedade Mundo Novo.

$$
\begin{aligned}
& E_{d-v e}=-22,750+5,266 \text { A } \quad\left(R^{2}=0,87\right) \\
& E_{d-v d}=-44,000+10,932 \text { A } \quad\left(R^{2}=0,89\right)
\end{aligned}
$$

A.

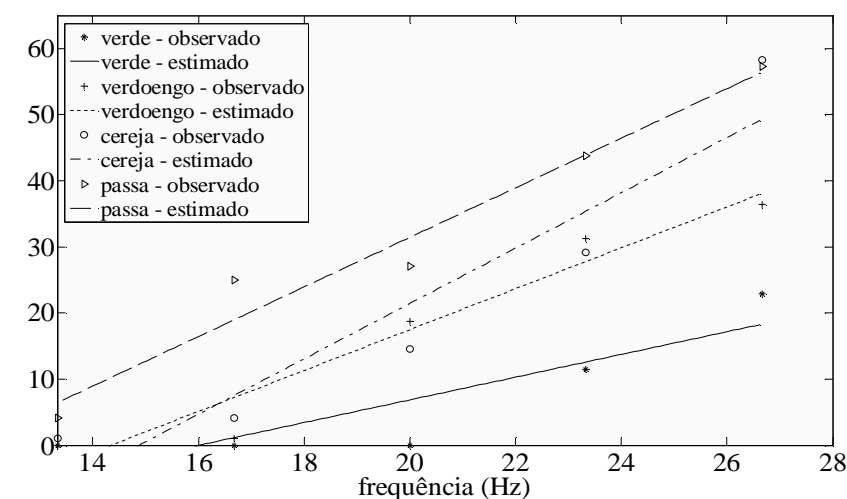

B.

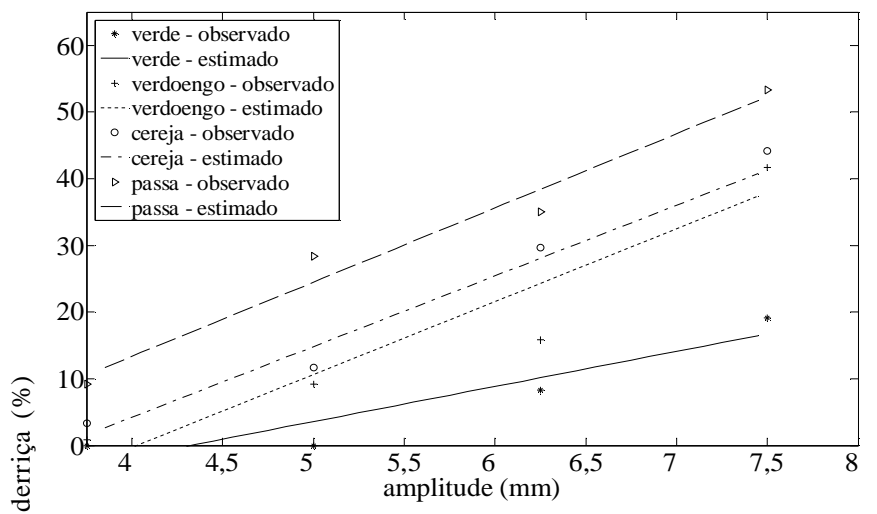

$\approx C$.

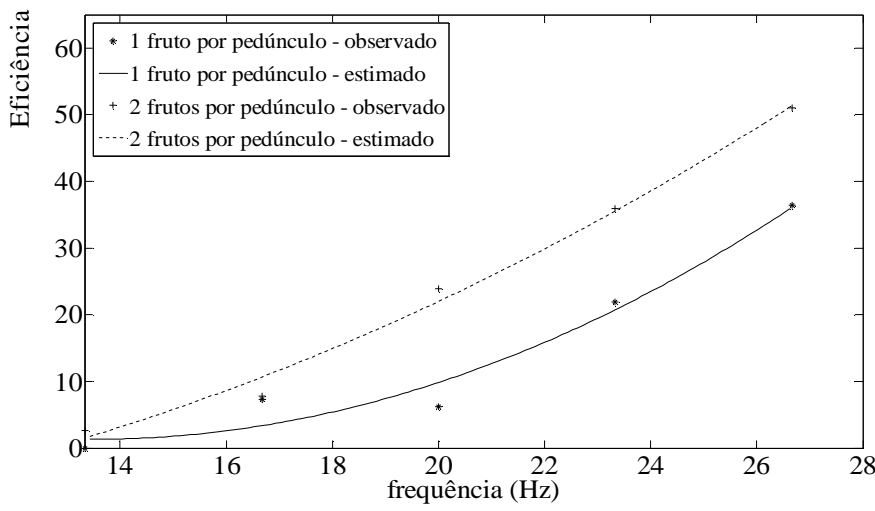

D.

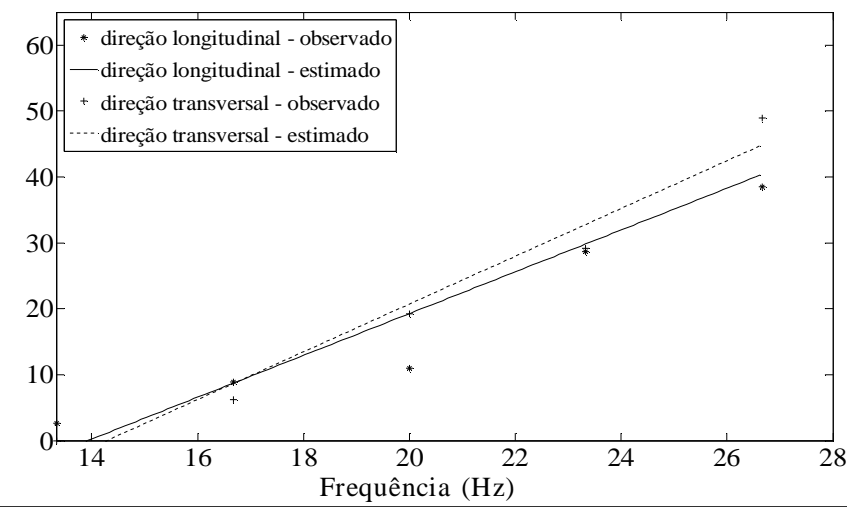

Figura 1. Efeito da frequência de vibração sob a eficiência de derriça nos diferentes graus de maturação $A$., efeito da amplitude de vibração sob a eficiência de derriça nos diferentes graus de maturação $B$., efeito da frequência de vibração sob a eficiência de derriça para diferentes números de frutos por pedúnculo $\mathrm{C}$. e efeito da frequência de vibração sob a eficiência de derriça para diferentes direções de vibração para a variedade Mundo N ovo D 


$$
\begin{array}{ll}
\mathrm{E}_{\mathrm{d}-\mathrm{ce}}=-38,166+10,600 \cdot \mathrm{A} & \left(\mathrm{R}^{2}=0,98\right) \\
\mathrm{E}_{\mathrm{d}-\mathrm{pa}}=-31,166+11,132 \cdot \mathrm{A} & \left(\mathrm{R}^{2}=0,97\right)
\end{array}
$$

em que:

$E_{d-v e}, E_{d-v d}, E_{d-c e}$ e $E_{d-p a}$ - eficiência de derriça para os graus de maturação verde, verdoengo, cereja e passa

$\mathrm{A}=$ amplitude de vibração

Tem-se, na Figura 1B, o efeito da amplitude de vibração em cada grau de maturação. Pode-se verificar que a eficiência de derriça aumenta na medida em que se aumenta a amplitude vibração. A amplitude de 7,5 mm mostrou uma eficiência maior de derriça, em todos os graus de maturação, o que pode ser explicado em função do aumento de energia ao qual o sistema fruto-pedúnculo se encontra submetido durante o procedimento de derriça.

Desta forma, a combinação adequada dos fatores frequência e amplitude de vibração é fundamental para o projeto de máquinas destinadas à colheita de café de forma eficiente. Ciro (2001) destaca que os fatores frequência, amplitude e tempo de vibração são essenciais para a colheita mecanizada de café por vibrações mecânicas, sendo a frequência de vibração um critério básico para o sucesso desse procedimento.

Apresentam-se, na Tabela 8, os resultados obtidos do desdobramento da interação entre os fatores amplitude e grau de maturação para a eficiência da derriça de ramos coletados na variedade Mundo Novo. Pode-se notar que a diferença significativa foi detectada entre graus de maturação, nas amplitudes de 5,0, 6,25 e 7,5 mm. As médias da eficiência da derriça entre os graus de maturação nessas amplitudes foram comparadas pelo teste Tukey a $5 \%$ de probabilidade e os resultados obtidos estão na Tabela 9 .

De acordo com a Tabela 9, para as amplitudes de 5,0 e 7,5 mm não se observaram diferenças significativas entre as mé-

Tabela 8. Resultados obtidos da análise de variância do desdobramento da interação entre os fatores amplitude e grau de maturação com relação a eficiência da derriça em ramos da

\begin{tabular}{|c|c|c|c|c|c|}
\hline FV & GL & SQ & QM & $\mathbf{F}$ & P-valor \\
\hline Maturação/amplitude (3,75 mm) & 3 & 3083,33 & 1027,77 & $1,42 \mathrm{~ns}$ & 0,2367 \\
\hline Maturação/amplitude (5,0 mm) & 3 & 25115,00 & 8371,52 & $11,53 *$ & $<0,001$ \\
\hline Maturação/amplitude (6,25 mm) & 3 & 26708,00 & 8902,77 & $12,26 *$ & $<0,001$ \\
\hline Maturação/amplitude (7,5mm) & 3 & 37875,00 & 12625,00 & $17,39 *$ & $<0,001$ \\
\hline Resíduo & 906 & 657750,00 & 725,99 & - & - \\
\hline
\end{tabular}
variedade Mundo Novo

* significativo a nível de $5 \%$ de probabilidade e ${ }^{\text {ns }}$ não-significativo

Tabela 9. Médias para eficiência de derriça em função da amplitude de vibração e do grau de maturação dos frutos, para a variedade Mundo Novo

\begin{tabular}{lcccc}
\hline \multicolumn{1}{c}{$\begin{array}{c}\text { Amplitude } \\
\text { (mm) }\end{array}$} & \multicolumn{4}{c}{ Grau de maturação } \\
\cline { 2 - 5 } & Verde & Verdoengo & Cereja & Passa \\
5,0 & $0,00 \mathrm{~A}$ & $9,17 \mathrm{~B}$ & $11,67 \mathrm{~B}$ & $28,33 \mathrm{C}$ \\
6,25 & $8,33 \mathrm{~A}$ & $15,83 \mathrm{~B}$ & $29,67 \mathrm{C}$ & $35,00 \mathrm{D}$ \\
7,5 & $19,17 \mathrm{~A}$ & $41,67 \mathrm{~B}$ & $44,17 \mathrm{~B}$ & $53,33 \mathrm{C}$ \\
\hline
\end{tabular}

Médias seguidas de letras iguais na mesma linha não diferem entre si, segundo o teste de Tukey, a probabilidade de $5 \%$ dias dos frutos verdoengos e cerejas; observou-se, contudo, que as médias dos frutos verdoengos e cerejas apresentaram diferenças significativas em relação às médias dos frutos verdes. Para amplitude de $6,25 \mathrm{~mm}$, as médias dos frutos verdes, verdoengo, cereja e passa apontaram diferenças significativas sendo a média dos frutos cereja consideravelmente superior à dos frutos verdes e verdoengos, o que é desejável para a realização da colheita seletiva de frutos do cafeeiro.

$\mathrm{Na}$ Tabela 10 se apresentam os resultados obtidos do desdobramento da interação entre os fatores frequência e número de frutos por pedúnculo, para a eficiência de derriça de ramos coletados na variedade Mundo Novo. As médias da eficiência de derriça entre os níveis do fator número de frutos por pedúnculo nessas frequências, foram comparadas pelo teste Tukey a $5 \%$ de probabilidade e os resultados obtidos se encontram na Tabela 11.

Tabela 10. Resultados obtidos da análise de variância do desdobramento da interação entre os fatores frequência $e$ número de frutos por pedúnculo com relação à eficiência da derriça, em ramos da variedade Mundo Novo

\begin{tabular}{lcrrrc}
\hline FV & GL & \multicolumn{1}{c}{ SQ } & \multicolumn{1}{c}{ QM } & F & P-valor \\
No de frutos/frequência $(13,33 \mathrm{~Hz})$ & 1 & 325,52 & 325,52 & $0,45^{\text {ns }}$ & 0,5033 \\
No de frutos/frequência $(16,67 \mathrm{~Hz})$ & 1 & 13,02 & 13,02 & $0,02^{\text {ns }}$ & 0,8935 \\
No de frutos/frequência $(20,00 \mathrm{~Hz})$ & 1 & 15052,00 & 15052,00 & $20,73^{*}$ & $<0,001$ \\
No de frutos/frequência $(23,33 \mathrm{~Hz})$ & 1 & 9492,19 & 9492,19 & $13,07^{*}$ & $<0,001$ \\
No de frutos/frequência $(26,67 \mathrm{~Hz})$ & 1 & 10208,00 & 10208,00 & $14,06^{*}$ & $<0,001$ \\
\multicolumn{1}{c}{ Resíduo } & 906 & 657750,00 & 725,99 & - & - \\
\hline
\end{tabular}

* significativo ao nível de $5 \%$ de probabilidade e ${ }^{\text {ns }}$ não-significativo

Tabela 11. Média da eficiência de derriça em função da frequência de excitação e do número de frutos por pedúnculo, em ramos coletados na variedade Mundo Novo

\begin{tabular}{ccc}
\hline Frequência $(\mathbf{H z})$ & Número de Frutos & Médias \\
\multirow{2}{*}{20,00} & 1 fruto & $6,65 \mathrm{~A}$ \\
& 2 frutos & $23,95 \mathrm{~B}$ \\
\hline \multirow{2}{*}{23,33} & 1 fruto & $21,87 \mathrm{~A}$ \\
& 2 frutos & $35,93 \mathrm{~B}$ \\
\hline \multirow{2}{*}{26,67} & 1 fruto & $36,45 \mathrm{~A}$ \\
& 2 frutos & $51,04 \mathrm{~B}$ \\
\hline
\end{tabular}

Médias seguidas de letras iguais para mesma frequência não diferem entre si segundo o teste de Tukey, a probabilidade de 5\%

De acordo com a Tabela 11, nas frequências de vibração 20,00, 23,33 e 26,67 Hz, pedúnculos com dois frutos apresentaram média de eficiência de derriça maior que pedúnculos com um fruto. A maior média de eficiência de derriça em pedúnculos com dois frutos, pode ser explicada devido às forças de interação e colisão ocorridas entre os dois frutos durante o período em que os ramos foram submetidos a vibração; em frequências de vibração menores que $20,00 \mathrm{~Hz}$ tal efeito parece não ocorrer (Tabela 10).

A Tabela 12 engloba o desdobramento do efeito da frequência de vibração para diferentes números de frutos por pedúnculo; tal estudo foi realizado por meio de regressão podendo-se observar que o efeito do fator frequência de vibração foi significativo tanto para um fruto por pedúnculo quanto para dois frutos por pedúnculo, o que novamente caracteriza 
Tabela 12. Resultados da análise de regressão para 0 estudo do efeito da frequência de vibração, considerando-se o número de frutos por pedúnculo na eficiência da derriça de ramos coletados na variedade $M$ undo Novo

\begin{tabular}{lcccc}
\hline \multirow{2}{*}{ FV } & \multicolumn{1}{c}{ 1 fruto por pedúnculo } & \multicolumn{2}{c}{ 2 frutos por pedúnculo } \\
\cline { 2 - 6 } & $\mathbf{G L}$ & $\mathbf{Q M}$ & $\mathbf{G L}$ & $\mathbf{Q M}$ \\
Regressão & 1 & $77198,68^{*}$ & 1 & $151657,21^{*}$ \\
Resíduo & 906 & 725,99 & 906 & 725,99 \\
\hline
\end{tabular}

* significativo ao nível de $5 \%$ de probabilidade

sua importância no procedimento de derriça por vibrações mecânicas.

As Eqs. 9 e 10 representam os modelos escolhidos com base no coeficiente de determinação e na significância dos coeficientes, para o estudo do efeito da amplitude de vibração em cada grau de maturação, para a variedade Mundo Novo.

$$
\begin{aligned}
& E_{d-1 f}=37,630-5,395 \cdot F+0,200 \quad F^{2}\left(R^{2}=0,94\right) \\
& E_{d-2 f}=-12,801-0,264 \cdot F+0,100 F^{2} \quad\left(R^{2}=0,98\right)
\end{aligned}
$$

em que:

$E_{d-1 f}$ e $E_{d-2 f}$ - eficiência de derriça para um e dois frutos por pedúnculo

Tem-se, na Figura 1C, o efeito da frequência de vibração sob a eficiência de derriça para um e dois frutos por pedúnculo nos ramos coletados na variedade Mundo Novo; pode-se observar que a frequência de vibração de $26,67 \mathrm{~Hz}$ apresentou uma eficiência maior de derriça, tanto para um quanto para dois frutos por pedúnculo; entretanto, pedúnculos com dois frutos apresentaram médias de eficiência de derriça superiores a pedúnculos com apenas um fruto.

Na Tabela 13 se apresenta o desdobramento do efeito da frequência de vibração para diferentes direções de vibração. $O$ efeito do fator frequência de vibração foi significativo tanto para a direção transversal quanto para a direção longitudinal de vibração.

Tabela 13. Resultado da análise de regressão para 0 estudo do efeito da frequência nas diferentes direções de vibração na eficiência de derriça de ramos coletados na variedade Mundo Novo

\begin{tabular}{lcccc}
\hline \multirow{2}{*}{ FV } & \multicolumn{2}{c}{ Direção Transversal } & \multicolumn{2}{c}{ Direção Longitudinal } \\
\cline { 2 - 5 } \multicolumn{1}{c}{ GL } & QM & GL & QM \\
Regressão & 1 & $140170,96 *$ & 1 & $107314,43^{*}$ \\
Resíduo & 906 & 725,99 & 906 & 725,99 \\
\hline
\end{tabular}

* significativo ao nível de $5 \%$ de probabilidade

As Eqs. 11 e 12 representam os modelos escolhidos para o estudo do efeito da frequência de vibração em cada direção de vibração para a variedade Mundo Novo.

$$
\begin{aligned}
& \mathrm{E}_{\mathrm{d}-\mathrm{rr}}=-51,757+3,624 \cdot \mathrm{F} \quad\left(\mathrm{R}^{2}=0,96\right) \\
& \mathrm{E}_{\mathrm{d}-\mathrm{lo}}=-44,153+3,171 \cdot \mathrm{F} \quad\left(\mathrm{R}^{2}=0,96\right)
\end{aligned}
$$

em que:

$E_{d-t r}$ e $E_{d-l o}$ - eficiência de derriça nas direções de vibração transversal e longitudinal

Pode-se verificar, conforme a Figura 1D, que a frequência de vibração de $26,67 \mathrm{~Hz}$ apresentou as maiores médias para eficiência de derriça, tanto para a direção transversal como para a direção longitudinal, o que caracteriza o fato de que, a medida em que se aumenta a frequência de vibração, aumenta-se também a energia fornecida ao sistema, o que promove aumento na eficiência de derriça dos frutos de café.

\section{CONCLUSÕES}

1. Os fatores frequência e amplitude de vibração apresentaram interação significativa para a variedade Mundo Novo.

2. Baixas frequências de vibração fizeram com que não houvesse diferença na média da eficiência de derriça; por outro lado, sempre que a frequência de vibração aumentou, também aumentou a média da eficiência de derriça dos frutos em seus diferentes graus de maturação.

3. O fator amplitude de vibração influenciou nas médias de eficiência de derriça; na medida em que as amplitudes de vibração foram aumentadas o mesmo se deu em relação à eficiência de derriça, em seus diferentes graus de maturação.

4. O efeito da frequência de vibração depende do número de frutos por pedúnculo. Pedúnculos com maior número de frutos tendem a apresentar maior média da eficiência de derriça.

\section{AGRADECIMENTOS}

Os autores do trabalho agradecem à FAPEMIG - Fundação de Amparo à Pesquisa do Estado de Minas Gerais, e ao Consórcio Brasileiro de Pesquisa e Desenvolvimento do Café, pelo apoio financeiro concedido em prol da realização desta pesquisa.

\section{LITERATURA CITADA}

Barbosa, J. A.; Salvador, N.; Silva, F. M. Desempenho operacional de derriçadoras mecânicas portáteis, em diferentes condições de lavouras cafeeiras. Revista Brasileira de Engenharia Agrícola e Ambiental, v.9, n.1, p.129-132, 2005.

Castro-García, S.; Blanco-Roldán, G. L.; Gil-Ribes, J. A.; Agüera-Vega, J. Dynamic analysis of olive trees in intensive orchards under forced vibration. Trees, v.22, p. 795-802, 2008.

Ciro, V. H. J. Coffee harvesting I: Determination of the natural frequencies of the fruit stem system in coffee trees. Applied Engineering in Agriculture, v.17, n.4, p.475-479, 2001.

Filgueiras, W. H. Modelagem da planta de café por elementos finitos para estudos de colheita por vibração. Viçosa: UFV, 2001. 81p. Dissertação Mestrado

Oliveira, E.; Silva, F. M.; Souza, Z. M.; Figueiredo, C. A. P. Influência da colheita mecanizada na produção cafeeira. Ciência Rural, v.37, n.5, p.1446-1470, 2007. 
Sessiz, A.; Özcan, M. T. Olive removal with pneumatic branch shaker and abscission chemical. Journal of Food Engineering, v.76, p.148-153, 2006.

Souza, C. M. A. Desenvolvimento e modelagem de sistemas de derriça e de abanação de frutos do cafeeiro. Viçosa: UFV, 2004. 123p. Tese Doutorado

Souza, C. M. A.; Queiroz, D. M. de; Pinto, F. de A. de C.; Corrêa, P. C. Derriça de frutos de café por vibração. Revista Brasileira de Armazenamento, v.27, p.32-37, 2002.
Souza, C. M. A.; Queiroz, D. M.; Rafull, L. Z. L. Derriçadora portátil na colheita total e seletiva de frutos do cafeeiro. Pesquisa Agropecuária Brasileira, v.41, n.11, p.1637-1642, 2006.

Srivastava, A. K.; Goering, C. E.; Rohrbach, R. P. Engineering principles of agricultural machines. Michigan: ASAE, 1996. 601p.

Tascón, C. E. O.; Mora, R. B.; Mejía, F. A.; Aristizábal-Tórres, I. D.; Gómez, C. A. R.; Uribe, J. R. S. Cosecha del café con vibradores portátiles del tallo. Revista Facultad Nacional de Agronomia de Medellin, v.58, n.1, p.2697-2708, 2005. 